\title{
K-ratio Measurements by WDS and EDS for Instrument Maintenance, Calibration, and Accurate Quantification
}

Heather Lowers ${ }^{1}$ and Paul Carpenter ${ }^{2}$

${ }^{1}$ U.S. Geological Survey, Denver, Colorado, United States, ${ }^{2}$ Washington University, St. Louis, Missouri, United States

Multispectrometer measurements used to increase precision of trace element analyses and combined WDS and EDS measurement to improve analysis efficiency are recent advances in microanalysis (e.g. Bullock 2019 and Goemann 2019). These techniques require mutual alignment of the WDS spectrometers in addition to correct instrument calibration and conversion to concentration units by the correction algorithm. A ratio of the measured $\mathrm{k}$-ratio ( $\left.\mathrm{k}_{\text {meas }}\right)$ to the theoretically calculated $\mathrm{k}$-ratio $\left(\mathrm{k}_{\text {calc }}\right.$ ) allows for evaluation of all instrument parameters and correction parameters. A ratio of unity $\left(\mathrm{k}_{\text {meas }} / \mathrm{k}_{\text {calc }}\right)$ demonstrates accuracy of the $\mathrm{k}_{\text {meas }}$, internal consistency of standards used, and confirmation of correction algorithm parameters. Agreement in this ratio among spectrometers validates the application of the advances mentioned above on a given instrument. A suite of natural and synthetic minerals and glasses were analyzed to determine this ratio as part of instrument acceptance at the USGS in Denver (USGS) and long-term maintenance protocols at Washington University in St. Louis (WU).

A series of CMAS glasses (Dalheim 1977) were used to verify spectrometer alignment on a newly installed JEOL 8530F Plus at the USGS. Data were collected from five spots on each glass at $15 \mathrm{kV}, 20 \mathrm{nA}$ probe current, 20 micron defocused beam diameter, and 30 seconds on the peak and background. The analytical protocols were set up so each element was measured simultaneously on as many spectrometers as possible. For example, Si was simultaneously measured on spectrometers one through five with crystals TAP, TAPL, PETL, PETL, and TAP, respectively, and on a Thermo Fisher $10 \mathrm{~mm}^{2}$ silicon drift detector. Primary standards were $\mathrm{MgO}, \mathrm{Al}_{2} \mathrm{O}_{3}, \mathrm{SiO}_{2}$, and $\mathrm{CaSiO}$. The multispectrometer $\mathrm{k}_{\text {meas }} / \mathrm{k}_{\text {calc }}$ values shown in Figure 1 demonstrate agreement within $+/-3 \%$ relative. The Al outliers on Figure 1 are from Dalheim Glass $\mathrm{E}$ which is known to have inaccuracies in the reported values.

Standards-based EDS analyses were acquired at $15 \mathrm{kV}, 25 \mathrm{nA}$ probe current, 20 micron defocused beam diameter, and 120 seconds live time with a $10 \mathrm{~mm}^{2}$ e2v Gresham silicon drift detector on the JEOL 8200 at WU. WDS analyses with off-peak background corrections were also acquired on the same instrument utilizing the same operating conditions. Primary standards used for calibration include $\mathrm{MgO}, \mathrm{Al}_{2} \mathrm{O}_{3}, \mathrm{SiO}_{2}$, $\mathrm{CaSiO}_{3}, \mathrm{TiO}_{2}$, and $\mathrm{Fe}_{2} \mathrm{O}_{3}$. Measurements were made on all spectrometers [e.g., 1-PET 3-PET/LiF 4PET/LiF 5-PETH/LiFH, 1-TAP, 2-TAP] on the following standards: spinel $\mathrm{MgAl}_{2} \mathrm{O}_{4}$, enstatite $\mathrm{MgSiO}_{3}$, forsterite $\mathrm{Mg}_{2} \mathrm{SiO}_{4}$, kyanite $\mathrm{Al}_{2} \mathrm{SiO}_{5}$, San Carlos fayalite $\mathrm{Fe}_{2} \mathrm{SiO}_{4}$, Springwater olivine $(\mathrm{Mg}, \mathrm{Fe})_{2} \mathrm{SiO}_{4}$, Natural Bridge diopside $\mathrm{CaMgSi}_{2} \mathrm{O}_{6}$, Alaska anorthite $\mathrm{CaAl}_{2} \mathrm{Si}_{2} \mathrm{O}_{8}$, Ilmen Mtns. ilmenite $\mathrm{FeTiO}_{3}$, titanite $\mathrm{CaTiSiO}_{5}$, and synthetic glasses in the CMAS and CMASF systems: Weill/Dalheim CMAS glasses, NBS $\mathrm{K} 411, \mathrm{~K} 412$. The $\mathrm{k}_{\text {meas }} / \mathrm{k}_{\text {calc }}$ values from both WDS and EDS demonstrate the accuracy and internal consistency of this suite of microprobe standards (Figure 2). Outliers are explained by measurement error, such as background error for the trace elements, but also incorrect accepted values for the concentration 
of the standard. There are incorrect values Dalheim glass $\mathrm{E}$ (green diamond above line at $\sim 10 \% \mathrm{Al}_{2} \mathrm{O}_{3}$ ), and Taylor titanite $\mathrm{Fe}_{2} \mathrm{O}_{3}$ value, which is wrong by a factor of 2 at $\sim 0.66 \mathrm{wt} \%$.

The data presented here show mutual agreement among WDS spectrometers and between the WDS spectrometers and EDS detector and demonstrate internal consistency of standards at both laboratories. It is suggested that these measurements be performed as part of long-term instrument maintenance and calibration in order to establish confidence that multispectrometer measurements used to improve trace element precision and combined WDS and EDS analyses are accurate techniques on a given instrument.

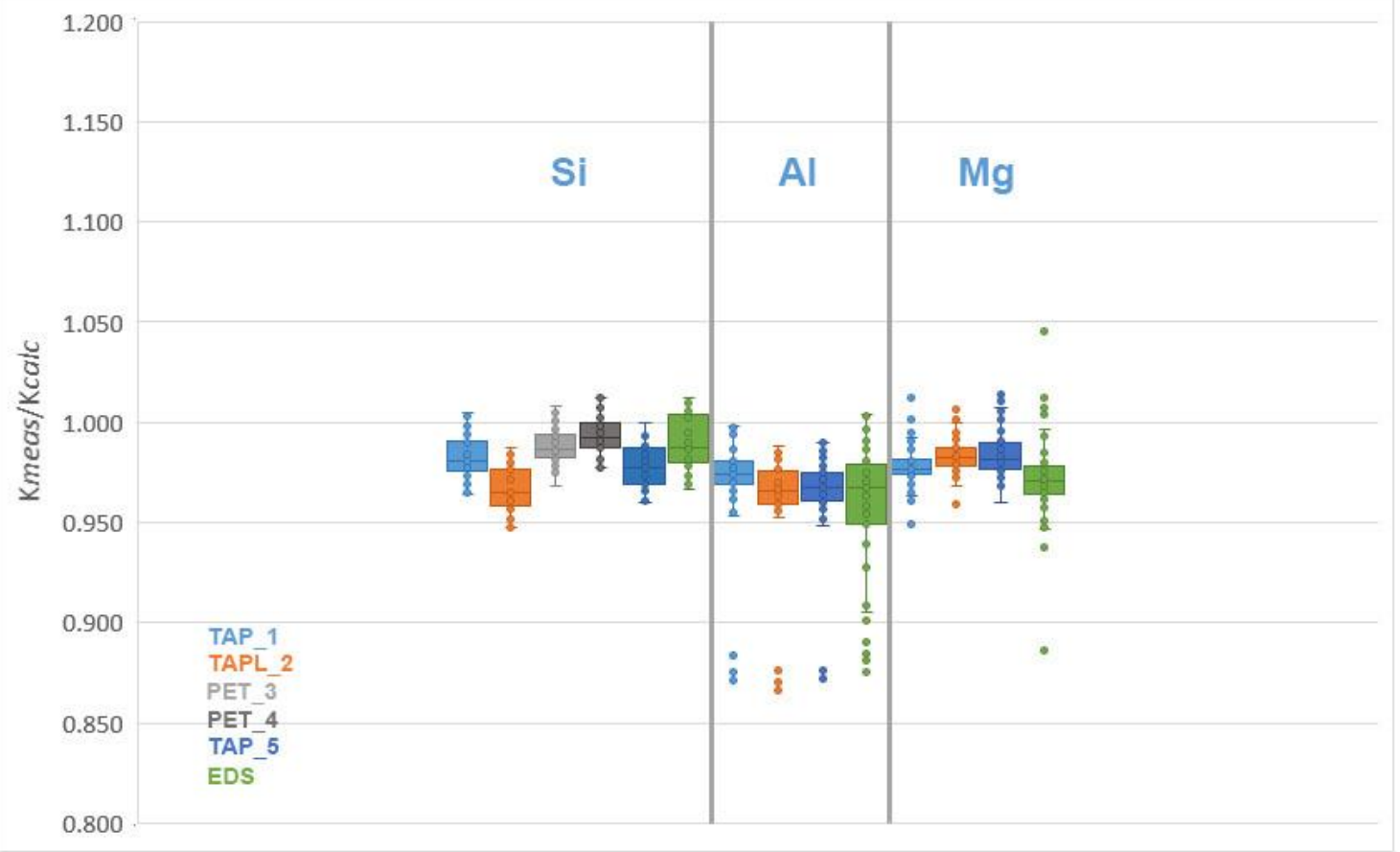

Figure 1. Multispectrometer Kmeas/Kcalc comparisons of $\mathrm{Si}, \mathrm{Al}$, and $\mathrm{Mg}$ measured in CMAS glasses. Each element was collected simultaneously on multiple WDS spectrometers and an EDS to confirm mutual agreement. Outliers are from a single glass (E) of questionable composition.
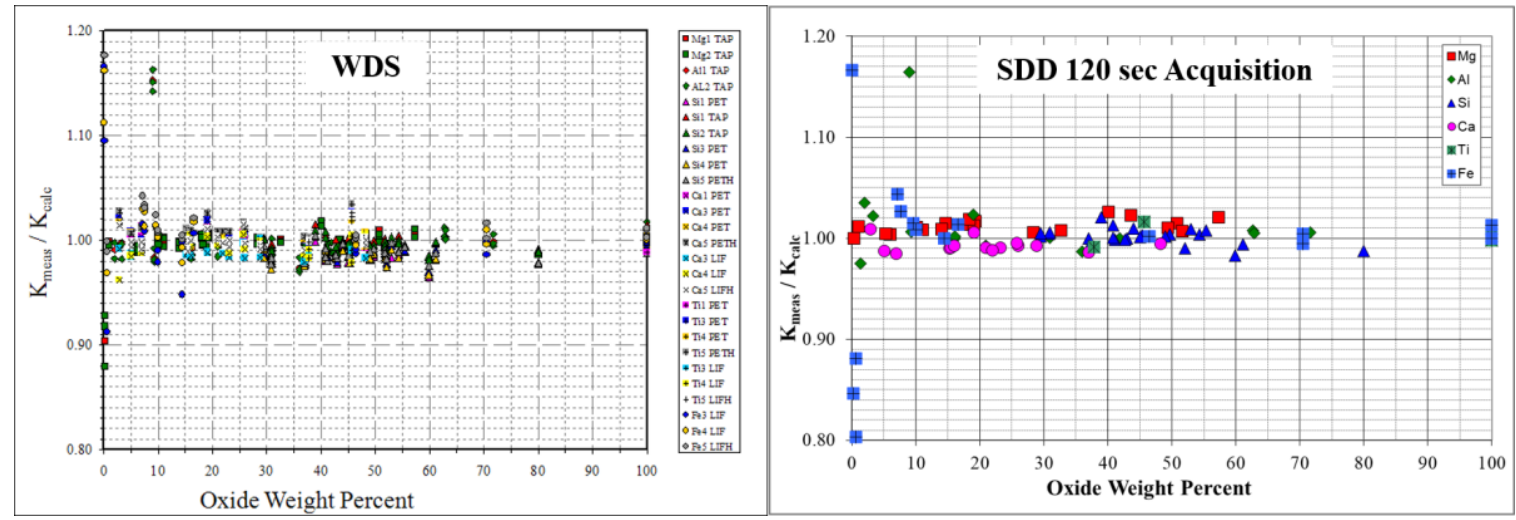

Figure 2. Kmeas/Kcalc from WDS measurements on left and EDS measurements on right demonstrate the expected accuracy, $+/-3 \%$ relative, from the techniques. Outliers are explained by measurement error or incorrect concentration of the accepted standard value. 


\section{References}

Bullock, E.S., 2019, Combined Energy Dispersive Spectrometry/Wavelength Dispersive Spectrometry Analysis of Basaltic Glass Using an Electron Probe Microanalyzer: Quantitative Microanalysis Topical Conference Program of Abstracts https://the-mas.org/wp-content/uploads/2019/06/QMA-2019-Programfinal-1.1-LQ.pdf

Dalheim, P.A., ms, 1977, Calculation of Empirical Coefficients in Multicomponent Oxide Systems for the Reduction of Electron Microprobe Data: M.S. thesis, University of Oregon, Eugene, Oregon.

Goemann, Karsten, 2019, Energy and Wavelength Dispersive X-ray Spectrometry in Electron Probe Microanalysis - The Best of Both Worlds is the Combination of the Two: Quantitative Microanalysis Topical Conference Program of Abstracts https://the-mas.org/wp-content/uploads/2019/06/QMA-2019Program-final-1.1-LQ.pdf 